\title{
POINTWISE IN TERMS OF WEAK CONVERGENCE ${ }^{1}$
}

\author{
J. R. BAXTER
}

ABSTRACT. Let $(\Omega, \mathcal{F}, \mu)$ be a measure space, $\mu(\Omega)<\infty$. Let $X_{n}$ be a sequence of measurable functions on $\Omega$ taking values in a compact metric space $M$. The set of bounded s:opping times $\tau$ for the $X_{n}$ is a directed set under the obvious ordering. The following theorem is proved: $X_{n}$ converges pointwise almost everywhere if and only if the generalized sequence $\int \phi\left(X_{\tau}\right) d \mu$ converges for every continuous function $\phi$ on $M$. The martingale theorem is proved as an application.

1. The general result. Let $(\Omega, \mathcal{F}, \mu)$ be a measure space, $\mu(\Omega)<\infty$. Let $M$ be a compact metric space, $\mathcal{C}(M)$ the space of continuous functions on $M$. Let $X_{n}=\Omega \rightarrow M$ be measurable, $n=1,2, \cdots$. Define $\mathcal{F}_{n}=\mathfrak{F}\left(X_{1}, \cdots, X_{n}\right)$. A map $\tau: \Omega \rightarrow\{1,2, \cdots\}$ will be called a stopping time if $\{\tau=n\} \in \mathcal{F}_{n}$ for all $n$. Let $\Gamma$ be the collection of bounded stopping times $\tau$.

Definition 1. A map $f: \Gamma \rightarrow \mathbf{R}$ will be called a generalized sequence. We write $f(\tau)=a_{\tau^{*}}$ A generalized sequence $a_{\tau}$ will be said to converge to a number $a$ if for every $\epsilon>0$ there exists $\sigma \in \Gamma$ such that $\left|a_{\tau}-a\right|<\epsilon$ for $\tau \geq \sigma$ (cf. [2, I.7.1]). Clearly we may choose $\sigma=n$ everywhere for some $n$. It is easy to see that a generalized sequence $a_{\tau}$ converges if and only if for every strictly increasing sequence $\tau(n) \in \Gamma$ the ordinary sequence $a_{\tau(n)}$ converges.

The basic result is Theorem 1. A similar theorem in a continuous-time setting appears in Meyer [3, Proposition 6(a), p. 232]. References to results of the same sort by F. Mertens and M. Rao are also given in [3].

Theorem 1. The following two statements are equivalent:

(i) $X_{n}$ converges pointwise almost everywhere on $\Omega$.

(ii) For every $\phi \in \mathcal{C}(M), \int \phi\left(X_{\tau}\right) d \mu$ is a convergent generalized sequence.

Proof. (i) $\Rightarrow$ (ii). Fix $\phi \in \mathcal{C}(M)$. If (i) holds then $\phi\left(X_{n}\right)$ converges pointwise almost everywhere on $\Omega$. The usual proof of Lebesgue's bounded convergence theorem applies without any real change.

Received by the editors July 15, 1973 and, in revised form, October 17, 1973. AMS (MOS) subject classifications (1970). Primary 60F 15, 60G45.

1 Supported by NSF GP-28683. 
(ii) $\Rightarrow$ (i). Let us first consider the case $M=[0,1]$. Let (ii) hold and (i) not hold. We will obtain a contradiction. Let $X^{-}=\lim \sup X_{n}, X_{-}=$ $\lim \inf X_{n}$. Then $\mu\left(\left\{X_{-} \neq X^{-}\right\}\right)>0$, so there exist $\alpha$ and $\beta$ such that $\mu\left(\left\{X_{-}<\alpha<\beta<X^{-}\right\}\right)>0$. Choose $\epsilon>0$ such that $\mu\left(\left\{X_{-}<\alpha<\beta<X^{-}\right\}\right)>\epsilon$. Define $\phi \in \mathcal{C}(M)$ so that $\phi=0$ on $[0, \alpha]$, and $\phi=1$ on $[\beta, 1]$. It is easy to see that for any $n$ we can find bounded stopping times $\sigma, \gamma$ such that $\gamma \geq \sigma \geq$ $n,\left\{X_{\sigma} \geq \alpha\right\} \subseteq\{\sigma=\gamma\}$, and $\mu\left(\left\{X_{\sigma}<\alpha<\beta<X_{\gamma}\right\}\right)>\epsilon$. Then $\int \phi\left(X_{\gamma}\right) \geq$ $\int \phi\left(X_{\sigma}\right)+\epsilon$. Hence the sequence $\int \phi\left(X_{\tau}\right)$ cannot converge, and a contradiction is obtained. Thus (ii) $\Rightarrow$ (i) is proved, for the case $M=[0,1]$. This is the standard case, since $[a, b]$ is homeomorphic to $[0,1]$ for $-\infty \leq a<b \leq \infty$.

In the general case, if (ii) holds we use the preceding result to show that $\phi\left(X_{n}\right)$ converges pointwise almost everywhere on $\Omega$, for each $\phi$ in $\mathcal{C}(M)$. Let $\left\{\phi_{i}\right\}$ be a countable dense subset of $\mathcal{C}(M)$. There exists $\Omega_{1} \subseteq \Omega$ such that $\mu\left(\Omega-\Omega_{1}\right)=0$ and $\phi_{i}\left(X_{n}(\omega)\right)$ converges as $n \rightarrow \infty$ for each $\omega \in \Omega_{1}$, and all $i$. Hence $\bar{\phi}\left(X_{n}(\omega)\right)$ converges as $n \rightarrow \infty$ for each $\omega$ in $\Omega_{1}$, and all $\phi$ in $\mathcal{C}(M)$. It follows that $X_{n}(\omega)$ converges for each $\omega$ in $\Omega_{1}$. This completes the proof of Theorem 1 .

Definition 2. A. collection $\mathcal{H} \subseteq \mathcal{C}(M)$ will be called a separating class if for any bounded Borel measures $p$ and $q$ on $M, \int \phi d p=\int \phi d q$ for all $\phi \in \mathcal{H}$ implies $p=q$. A collection $\mathcal{K}$ of Borel functions on $M$ will be said to generate a separating class if the set of all linear combinations of functions in $\mathcal{K}$ contains a separating class in $\mathcal{C}(M)$. The functions in $\mathcal{K}$ need not be continuous.

Corollary to Theorem 1. The following condition is equivalent to (i) and (ii) of Theorem 1:

(iii) $\int \phi\left(X_{\tau}\right) d \mu$ exists and is convergent for each $\phi$ in a collection $\mathcal{K}$ that generates a separating class in $\mathcal{C}(M)$.

Proof. It is enough to show (iii) $\Rightarrow$ (ii). We may assume $\mathcal{K}$ is a separating class in $\mathcal{C}(M)$. The proof is then the same as that for ordinary sequences, applied to $X_{\tau(n)}$ for each strictly increasing sequence $\tau(n) \in \Gamma$.

Example 1. Let $M=[-\infty, \infty]$. Let $\mathcal{K}_{0}$ be the collection of functions $\phi$, continuous on $(-\infty, \infty)$, such that:

(1) $\phi$ is constant on $(-\infty, a]$ and $\phi$ is linear and increasing on $[a, \infty)$, for some $a$, and

(2) $\phi$ is arbitrary at $\pm \infty$.

Then $\mathcal{K}_{0}$ generates a separating class in $\mathcal{C}(M)$, since the characteristic function of any interval $[b, \infty)$ is a bounded pointwise limit of differences of members of $\mathcal{K}_{0}$. 
It is clearly desirable to have to check the convergence of $\int \phi\left(X_{\tau}\right) d \mu$ for as few $\phi$ 's as possible. The following observation is useful in this respect, though it will not be needed in $\$ 2$.

Lemma. Let $\phi$ and $\theta$ be Borel functions on $M$ such that $\int \phi\left(X_{\tau}\right) d \mu$ and $\int \theta\left(X_{\tau}\right) d \mu$ converge and such that $\int \phi \vee \theta\left(X_{\tau}\right) d \mu$ is bounded above. Then $\int \phi \vee \theta\left(X_{\tau}\right) d \mu$ converges.

Proof. Given $\epsilon>0$, choose $N$ such that $\tau, \sigma \geq N$ implies $\mid \int \phi\left(X_{\tau}\right)-$ $\int \phi\left(X_{\sigma}\right) \mid<\epsilon$ and $\left|\int \theta\left(X_{\tau}\right)-\int \theta\left(X_{\sigma}\right)\right|<\epsilon$. Choose $\tau \geq N$ such that $\int \phi \vee \theta\left(X_{\sigma}\right)<$ $\int \phi \vee \theta\left(X_{\tau}\right)+\epsilon$ for any $\sigma \geq N$. Now suppose $\sigma \geq \tau$. Choose Borel sets $A$ and $B$ such that $A \cap B=\varnothing, A \cup B=M, \phi \vee \theta=\phi$ on $A$, and $\phi \vee \theta=\theta$ on $B$. Define the stopping time $\sigma_{1}$ by $\sigma_{1}=\sigma$ on $X_{\tau}^{-1}(A)$ and $\sigma_{1}=\tau$ on $X_{\tau}^{-1}(B)$. Clearly $\int \phi\left(X_{\tau}\right)<\int \phi\left(X_{\sigma_{1}}\right)+\epsilon_{\text {. Then }}$

$$
\int_{X_{\tau}^{-1}(A)} \phi \vee \theta\left(X_{\tau}\right)<\int_{X_{\tau}^{-1}(A)} \phi \vee \theta\left(X_{\sigma}\right)+\epsilon .
$$

Similarly

$$
\int_{X_{\tau}^{-1}(B)} \phi \vee \theta\left(X_{\tau}\right)<\int_{X_{\tau}^{-1}(B)} \phi \vee \theta\left(X_{\sigma}\right)+\epsilon .
$$

Thus $\int \phi \vee \theta\left(X_{\tau}\right)<\int \phi \vee \theta\left(X_{\sigma}\right)+2 \epsilon$, so that

$$
\left|\int \phi \vee \theta\left(x_{\tau}\right)-\int \phi \vee \theta\left(x_{\sigma}\right)\right|<2 \epsilon
$$

and the Lemma is proved.

2. The martingale theorem. Let $M=[-\infty, \infty]$. The sequence $X_{n}$ of $\S_{1}$ will be called a submartingale if

(a) $E\left(\left|X_{n}\right|\right)<\infty$ for all $n$, and

(b) $E\left(X_{n+1} \mid X_{n}, \cdots, X_{1}\right) \geq X_{n}$ for all $n$.

We wish to prove the martingale theorem [1, Chapter 7$]$ :

Theorem 2. Let $E\left(\left|X_{n}\right|\right)$ be bounded. Then $X_{n}$ converges pointwise almost everywhere.

Proof. (a) and (b) imply by the submartingale stopping the orem that for $\tau, \sigma \in \Gamma$ with $\tau \geq \sigma$ we have $E\left(X_{\tau} \mid X_{\sigma}\right) \geq X_{\sigma^{*}}$. Then by Jensen's inequality we have $E\left(\phi\left(X_{\tau}\right)\right) \geq E\left(\phi\left(X_{\sigma}\right)\right)$ for any $\phi \in \mathcal{K}_{0}$, where $\mathcal{K}_{0}$ is defined in Example 1. That is, $\int \phi\left(X_{\tau}\right) d \mu$ is a monotonic generalized sequence for each $\phi \in \mathcal{K}_{0}$. Since $\int\left|X_{n}\right| d \mu$ is bounded, so is $\int \phi\left(X_{n}\right) d \mu$, for each $\phi \in \mathcal{K}_{0}$. Hence, since $\int \phi\left(X_{\tau}\right) d \mu$ is monotonic, it is also bounded, for each $\phi \in K_{0^{*}}$. As usual a 
bounded, monotonic generalized sequence must converge. By the corollary to Theorem 1, $X_{n}$ must converge almost everywhere on $\Omega$ to a finite or infinite limit. The boundedness of $E\left(\left|X_{n}\right|\right)$ shows that the limit is finite almost everywhere, so Theorem 2 is proved.

The author wishes to thank Professor R. V. Chacon for stimulating and useful discussions on this topic.

\section{REFERENCES}

1. J. L. Doob, Stochastic processes, Wiley, New York; Chapman \& Hall, London, 1953. MR 15, 445.

2. N. Dunford and J. T. Schwartz, Linear operators. I: General theory, Pure and Appl. Math., vol. 7, Interscience, New York, 1958. MR 22 \#8302.

3. P. A. Meyer, Le retournement du temps, d'apres Chung et Walsh, Séminaire de Probabilités V, Univ. de Strasbourg, Lecture Notes in Math., vol. 191, SpringerVerlag, New York, 1971.

DEPARTMENT OF MATHEMATICS, UNIVERSITY OF MINNESOTA, MINNEA POLIS, MINNESOTA 55455 